\title{
The role of ionic vs. non-ionic excipients in APIs-based eutectic systems
}

\author{
Mónia A.R. Martins ${ }^{\mathrm{a}}$, Liliana P. Silva ${ }^{\mathrm{a}}$, Patrícia S. Jorge ${ }^{\mathrm{a}}$, Dinis O. Abranches ${ }^{\mathrm{a}}$, Simão P. Pinho ${ }^{\mathrm{b}}$, \\ João A.P. Coutinho ${ }^{\mathrm{a}, *}$ \\ ${ }^{\text {a }}$ CICECO - Aveiro Institute of Materials, Department of Chemistry, University of Aveiro, 3810-193 Aveiro, Portugal \\ ${ }^{\mathrm{b}}$ Mountain Research Center - CIMO, Polytechnic Institute of Bragança, 5301-855 Bragança, Portugal
}

\section{A R T I C L E I N F O}

\section{Keywords:}

Drug formulation

Thymol

Quaternary ammonium chlorides

Solid-liquid equilibrium

Phase diagrams

COSMO-RS

\begin{abstract}
A B S T R A C T
Aiming to contribute to drug pre-formulation, new eutectic mixtures were developed. Thymol, coumarin, or quaternary ammonium chlorides as excipients, were combined with the active pharmaceutical ingredients (APIs) acetylsalicylic acid, acetaminophen, ibuprofen, ketoprofen, or lidocaine. Their solid-liquid equilibrium (SLE) binary phase diagrams were measured to study eventual phase separation between the compounds, preventing manufacturing problems, and to study the molecular interactions between the APIs and ionic or non-ionic excipients. The Conductor-like Screening Model for Real Solvents (COSMO-RS) capability to predict the SLE of mixtures containing non-ionic excipients was further evaluated. COSMO-RS gives a good quantitative description of the experimental SLE being a tool with great potential in the screening of eutectic systems containing APIs and non-ionic excipients. While thymol presents strong interactions with the APIs, and consequently negative deviations to thermodynamic ideality, systems containing coumarin follow a quasi-ideal behavior. Regarding the ionic excipients, both choline chloride and the tetraalkylammonium chlorides are unable to establish relevant interactions with the APIs, and no significant negative deviations to ideality are observed. The liquefaction of the APIs here studied is favored by using non-ionic excipients, such as thymol, due to the strong interactions it can establish with the APIs.
\end{abstract}

\section{Introduction}

Pharmaceuticals are an essential element in medicine, and due to their benefits to society, their demand increases every day. However, the development of new drugs is expensive and time-consuming. On average, around 10 years and more than 800 million dollars are required to design and test a new drug (Berg et al., 2010; Tiwari et al., 2012). Clinical development that comprises pre-clinical research and clinical trials are the main issues to be accounted for, but it is also important to consider the challenges with drug discovery or product development. The search for the best excipient is related to chemical instability, poor water solubility, crystallinity, and polymorphism (Strickley and Oliyai, 2007). More than $40 \%$ of the drugs under development are classified as poorly soluble, or even insoluble, and to improve their bioavailability they should be in the liquid state or sufficiently soluble in the gastric fluids at the absorption point (Kalepu and Nekkanti, 2015; Savjani et al., 2012). Regarding dermal drug delivery, problems arise from physicochemical restrictions imposed by the protective function of human skin. Key factors for a drug to be delivered passively via the skin are suitable lipophilicity and molecular weight
(Brown et al., 2006). Thus, approaches that would increase the bioavailability of the compounds leading to lower dosages and decreasing the wastage would be of importance.

Improving the formulation of existing drugs is much simpler and faster than the development of new ones. Therefore, many attempts to find solutions for that particular challenge faced by the pharmaceutical industry have been followed (Kalepu and Nekkanti, 2015; Pedro et al., 2019). Examples comprise chemical modifications such as changing the solution $\mathrm{pH}$, derivatization or the use of buffers, and/or physical changes such as particle size reduction, solid dispersions, and drug dispersion by eutectic formation (Savjani et al., 2012).

The formation of eutectic mixtures, and more recently deep eutectic mixtures or deep eutectic solvents (DES), has been proposed as a technique to increase active pharmaceutical ingredients (APIs) water solubility and bioavailability on the development of new drugs (Cherukuvada and Nangia, 2014; Gala et al., 2013; Martins et al., 2019; Pedro et al., 2019; Savjani et al., 2012). These mixtures are characterized by a decrease in the melting temperature of the system compared to the pure components melting temperatures, while keeping the therapeutic integrity of the forming compounds. Eutectic mixtures

\footnotetext{
* Corresponding author..

E-mail address: jcoutinho@ua.pt (J.A.P. Coutinho).
} 
are expected to have a significant role in the pharmaceutical industry if they are formed between APIs, between APIs and excipient, or between excipients, where excipients are defined as molecules with the ability to deliver the active ingredient to the active site (Gala et al., 2013; Kalasz and Antal, 2006). The choice of the appropriate excipients and APIs, and their respective ratios, are crucial during drug pre-formulation. Wrong choices either in terms of composition or temperature range at which mixtures are at a given physical state, may lead to the undesirable melting of a pharmaceutical tablet or even decomposition of the pure components (Gala et al., 2013; Pedro et al., 2019). Thus, it is essential to know the solid-liquid equilibria (SLE) phase diagrams of the mixtures in order to understand the range of temperatures and compositions at which these mixtures can be used and stored (Sangster, 1999).

Aiming to increase the solubility and bioavailability of APIs, terpenes, and in particular menthol and thymol, have been used as excipients in a variety of works involving eutectics in the pharmaceutical industry (Cherukuvada and Nangia, 2014; Gala et al., 2013; Mohammadi-Samani et al., 2014; Stott et al., 1998) giving rise to some patents (Jun and Kang, 1999; Schwarz and Weisspapir, 2005). Stott et al. (1998) studied mixtures between ibuprofen, a well-known anti-inflammatory drug, and terpenes such as L-menthol or thymol. The authors reported that the eutectic temperature of thymol and ibuprofen was $305.15 \mathrm{~K}$ for $40: 60 \% \mathrm{w} / \mathrm{w}$ and that the solubility of this mixture is enhanced by 12.7 -fold over the saturated aqueous solution of ibuprofen (Stott et al., 1998). The observed melting point depression led to a faster transdermal penetration. A similar effect was observed for the mixture of meloxicam and thymol, where the terpenoid is a skin permeation enhancer that increases the meloxicam skin absorption (Mohammadi-Samani et al., 2014). Other authors (Abbott et al., 2017; Aroso et al., 2016; Duarte et al., 2017) combined menthol and/or choline chloride, $[\mathrm{Ch}] \mathrm{Cl}$, with different APIs such as ibuprofen, acetaminophen, or acetylsalicylic acid. These combinations were reported to liquefy close to body temperature $(310.15 \mathrm{~K})$, improving the API bioavailability (Aroso et al., 2016; Duarte et al., 2017). Abranches et al. (2019a) used the COSMO-RS model to predict the SLE of systems composed by $[\mathrm{Ch}] \mathrm{Cl}$ and APIs obtaining a reasonable description of the systems studied.

Choline chloride is widely used to prepare deep eutectic systems, i.e., eutectic mixtures that present negative deviations to ideality due to strong intermolecular interactions established between their components (Martins et al., 2019). However, the ability of [Ch]Cl to form mixtures with sharp melting temperature depressions (sometimes even liquid at room temperature) is mainly due to its low melting enthalpy, and not particularly due to strong interactions with other compounds (Fernandez et al., 2017). Moreover, its generalized use is due to its price, low toxicity, biocompatibility and biodegradability, and it was also studied as a possible pharmaceutical ingredient (Abbott et al., 2017; Abranches et al., 2019a). Recently, we have also investigated other quaternary ammonium chlorides (Abranches et al., 2020; Pontes et al., 2017) and surprisingly found them as equally successful, or even better ionic liquefying agents, to use in the design of eutectic mixtures (Abranches et al., 2020).

Thymol and coumarin were selected as non-ionic excipients (structures available in Table 1) due to their characteristics: while thymol is a stronger than usual hydrogen bond donor (and a weaker than usual hydrogen bond acceptor), coumarin is a simple hydrogen bond acceptor (Abranches et al., 2019b; Abranches et al., 2020), providing an interesting contrast in terms of interactions with the APIs. According to Abranches et al. (2019b) these non-ionic mixtures can form type V DES, a new class considering the original classification of DES proposed by Abbott and co-workers (Smith et al., 2014). Three quaternary ammonium salts were chosen as ionic excipients due to their proven success as liquefying agents (Abranches et al., 2020). Choline chloride, tetramethylammonium chloride, and tetrabutylammonium chloride were selected to study both the effect of the choline hydroxyl group and the increase of the alkyl chain length of the ammonium cation that leads to a substantial decrease in the compound melting point. Mixtures of APIs and quaternary ammonium salts are classified as type III DES: quaternary ammonium salt + hydrogen bond donor (Smith et al., 2014).

Aiming to contribute to the development of new drug formulations, binary eutectic mixtures involving thymol, coumarin, $[\mathrm{Ch}] \mathrm{Cl}$, tetramethylammonium chloride $\left(\left[\mathrm{N}_{1111}\right] \mathrm{Cl}\right)$ or tetrabutylammonium chloride $\left(\left[\mathrm{N}_{4444}\right] \mathrm{Cl}\right)$ and one API among acetylsalicylic acid, ibuprofen, ketoprofen, lidocaine or acetaminophen are here investigated. The choice of the APIs was based on their use in the pharmaceutical industry, functional groups diversity and number, low melting temperatures, and no decomposition upon melting. The solid-liquid phase diagrams were measured to characterize these mixtures, and the main goal was to explore the impact of the excipient nature (ionic or non-ionic) on the eutectic formation of the mixtures containing APIs. The ability of the conductor-like screening model for real solvents - COSMO-RS to describe the SLE phase diagrams of mixtures involving non-ionic excipients was also evaluated.

\section{Experimental}

\subsection{Chemicals}

Table 1 summarizes the information of the compounds investigated alongside with their structures. It also includes the melting temperatures and enthalpies measured in this work or obtained from the literature. All chemicals were used as received from the supplier without any further purification excepting the quaternary ammonium salts that were dried under vacuum $(0.1 \mathrm{~Pa}$ and $298.15 \mathrm{~K})$ for at least $72 \mathrm{~h}$. The water content of all compounds was measured using a Metrohm 831 Karl-Fischer coulometer, with the analyte Hydranal ${ }^{\oplus}-$ Coulomat AG from Riedel-de Haën, and is reported in Table 1.

\subsection{Phase diagrams measurements}

Binary mixtures between excipients and APIs were prepared in different proportions covering the full compositions range (at mole fraction intervals of 0.1 ) by weighting the proper amounts of each pure substance using an analytic balance Mettler Toledo XP205 (repeatability of $0.015 \mathrm{mg}$ ). Mixtures involving ionic excipients were prepared inside a dry-argon glove-box using an analytical balance (model ALS 220-4 N from Kern, with a repeatability of $0.2 \mathrm{mg}$ ).

Mixtures comprising thymol + acetylsalicylic acid, ibuprofen, ketoprofen, or acetaminophen and coumarin + ibuprofen were prepared by homogenizing the mixture of API and excipient with a mortar and pestle. Remaining mixtures were heated under stirring in closed vials till a homogeneous liquid was formed and left under stirring for around 15 minutes. As a final preparation step, the flasks were left at room temperature for 2-3 days in order to check for possible phase separation, indicating incompatibilities between the compounds. Depending on the physical state of the final mixture obtained (solid, past-like consistency, or liquid), the eutectic and melting temperatures, i.e., the solidus and the liquidus lines were determined using a melting point apparatus, differential scanning calorimetry, or both techniques as described below.

Melting point device: The obtained solid mixtures were transferred into a capillary. The melting temperatures were determined with an automatic glass capillary device model M-565 from Buchi (temperature

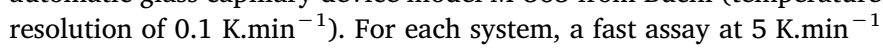
was initially carried out. Then the measurement was repeated at least three times applying a heating rate of 1 or $2 \mathrm{~K} \cdot \mathrm{min}^{-1}$. The eutectic temperature was registered when the first liquid was observed inside the capillary, and the melting temperature was taken when the last solid disappears. The melting temperatures of the pure components were also measured using this technique. 
Table 1

List of the studied compounds, chemical structures and melting properties (melting temperature $-T_{m}$ and enthalpy $-\Delta_{m} H$ ).

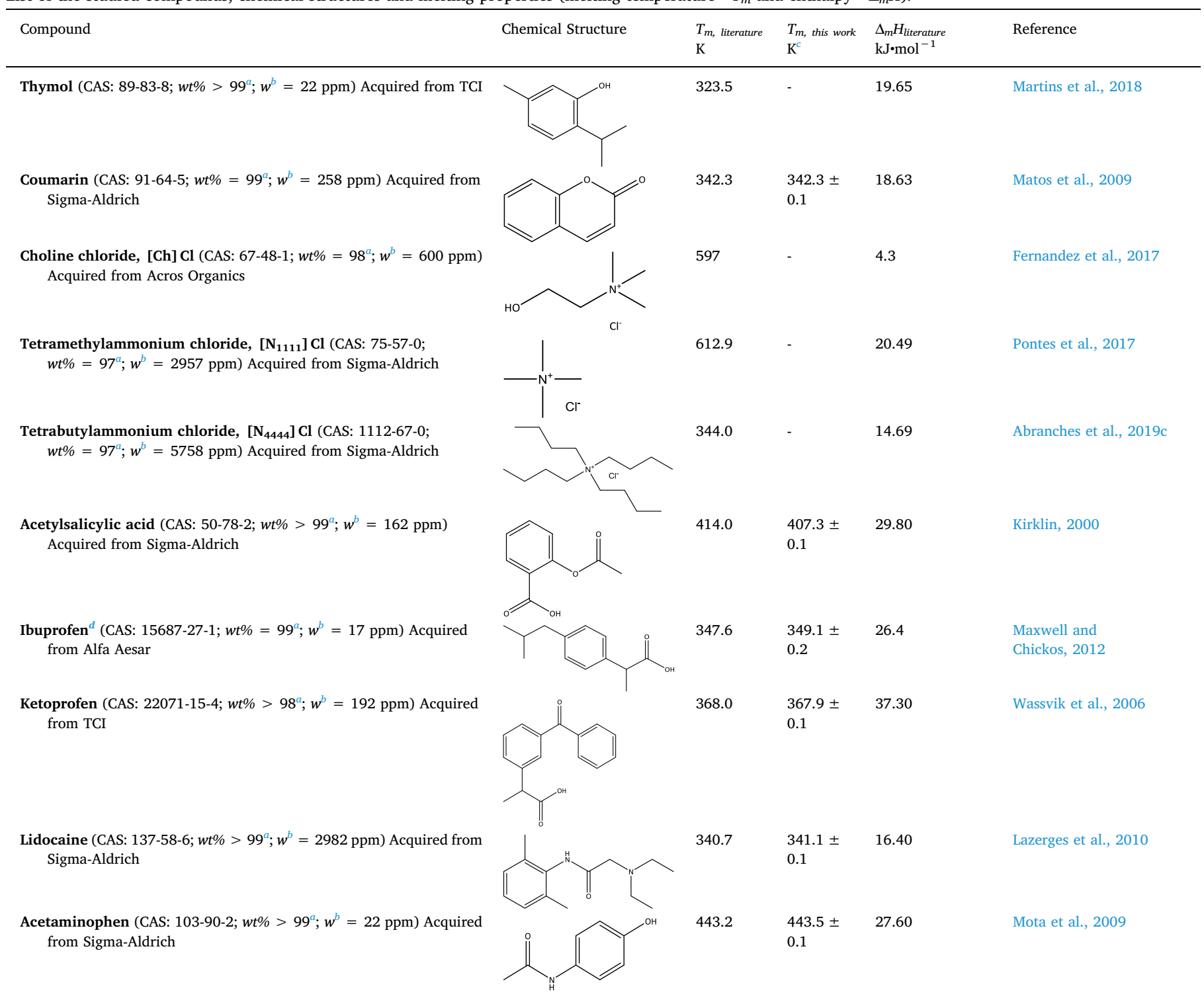

\footnotetext{
a As reported by the supplier;

b Water content;

c Measured in this work using a glass capillary visual method;

d Racemic form.
}

Differential Scanning Calorimetry (DSC): The melting temperatures of past-like consistency or liquid mixtures were determined using a Hitachi DSC7000X working at atmospheric pressure and coupled to a liquid nitrogen cooling system. Samples of approximately $5 \mathrm{mg}$ were weighted in a micro-analytical balance AD6 (PerkinElmer, precision $=0.002 \mathrm{mg})$ and then sealed in hermetic aluminum crucibles $(0.3$ $\mathrm{MPa}, 7.5 \mu \mathrm{L}$ ). Cooling and heating cycles at 5 and $2 \mathrm{~K} \mathrm{~min}^{-1}$, respectively, were performed. The temperature ranged from $223.15 \mathrm{~K}$ to $353.15 \mathrm{~K}$ for both systems investigated using DSC (coumarin + lidocaine and thymol + lidocaine). Thermal transitions were taken as the peak temperature. The equipment was previously calibrated with several standards (heptane, octane, decane, 4-nitrotoluene, naphthalene, benzoic acid, diphenylacetic acid, indium, tin, caffeine, lead, zinc, potassium nitrate, water, and anthracene) presenting weight fraction purities higher than $99 \%$.

\section{Modelling}

\subsection{Solid-liquid equilibrium}

The melting curves of each individual component in a binary mixture can be described by the simplified solid-liquid equilibrium equation (Prausnitz et al., 1970):

$\ln \left(x_{i} \gamma_{i}^{l}\right)=\frac{\Delta_{m} H}{R}\left(\frac{1}{T_{m}}-\frac{1}{T}\right)+\frac{\Delta_{m} C_{p}}{R}\left(\frac{T_{m}}{T}-\ln \frac{T_{m}}{T}-1\right)$

where the activity coefficient of compound $i$ at a certain liquid mole fraction composition $x_{i}$ is denoted as $\gamma_{i}^{l}, T$ is the absolute temperature, $T_{\mathrm{m}}$ and $\Delta_{\mathrm{m}} H$ are the melting temperature and enthalpy of the pure compound, respectively, $R$ is the universal gas constant, and $\Delta_{\mathrm{m}} C_{p}$ is the 
difference between the molar heat capacity of compound $i$ in the liquid and solid states. This equation assumes pure solid phases and neglects the temperature influence on heat capacities. In some cases, especially when a small difference between the equilibrium temperature and the melting temperature of the pure compounds is verified, the second term of the equation has a negligible value when compared to the first term. Consequently, it can be neglected (Coutinho et al., 1995; Elliott and Lira, 2012), due to its small contribution to the phase equilibrium calculations (Eq. (2)).

$\ln \left(x_{i} \gamma_{i}^{l}\right)=\frac{\Delta_{m} H}{R}\left(\frac{1}{T_{m}}-\frac{1}{T}\right)$

When an ideal liquid phase is assumed the activity coefficients $\left(\gamma_{i}^{l}\right)$ are equal to one and the solubility curves can be obtained from Eq. (2) as a function of the melting properties of the pure compounds. On the other hand, the experimental activity coefficients can be obtained as well from Eq. (2) using the experimental SLE data (Prausnitz et al., 1970).

\subsection{COSMO-RS}

The conductor-like screening model for real solvents (COSMO-RS) is a continuum solvent model for the prediction of the phase behavior of mixtures. The calculation principle is based on the combination of quantum chemistry and statistical thermodynamics (Klamt, 2005). The SLE phase diagrams were modelled with the software COSMOtherm (COSMOtherm, Release 19; COSMOlogic GmbH \& Co. KG, http://www. cosmologic.de.cosmotherm, Release 19; COSMOlogic GmbH \& Co. KG, http://www.cosmologic.de; Eckert and Klamt, 2002) with the parametrization BP_TZVP_19.ctd. The input $\sigma$ profiles were prepared using TURBOMOLE with the COSMO-BP-TZVP template of the software package TmoleX (TURBOMOLE V7.1, 2016, a development of University of Karlsruhe and Forschungszentrum Karlsruhe GmbH, 19892007, TURBOMOLE GmbH, since 2007; available from http://www. turbomole.com). This template consists of a def-TZVP basis set, a DFT with the B-P86 functional level of theory, and the COSMO solvent model (infinite permittivity).

\section{Results and discussion}

The appearance of a liquid phase from a mixture between two solid compounds results from the melting point depression (compared to the pure compounds melting points) observed in the solid-liquid phase diagram, which is dependent on the mixture composition, the melting properties of the pure components as well as the interactions established between them. For a system to present negative deviations from ideality, these interactions must be more intense than the interactions the pure compounds present in their (even if hypothetical) liquid state. The greater the negative deviations to ideality are, the larger the temperature depression. Mixtures showing significant negative deviations to thermodynamic ideality may be called deep eutectic systems (Martins et al., 2019).

In the following, the impact of ionic and non-ionic excipients in mixtures containing well-known APIs was investigated through their solid-liquid phase diagrams. The main goal is to understand the interactions between the mixture components, allowing to choose the appropriate substance to decrease the melting temperature (and even liquefy) an API.

\subsection{Non-ionic excipients}

The measured SLE phase diagrams of mixtures between thymol or coumarin, and the APIs acetylsalicylic acid, ibuprofen, ketoprofen, lidocaine, or acetaminophen are shown in Fig. 1 (detailed data listed in Tables S1 and S2 in the supporting information). The ideal solubility curves $\left(\gamma_{i}^{l}=1\right)$, calculated applying the measured melting temperatures and the enthalpies from literature (Table 1), are also shown. The experimental activity coefficients were calculated by Eq. (2) and listed in SI (Table S1 and Figure S1). Furthermore, the phase diagrams include the solidus line whenever these points were possible to measure. The predictive model COSMO-RS was further used to describe the phase behavior of these binary systems, and the results are also presented in Fig. 1.

The differences between the experimental melting temperatures measured in this work for pure compounds - Table 1 - and those from literature are less than $1.5 \mathrm{~K}$, validating the experimental methodology used. Acetylsalicylic acid is an exception, where this difference is of 6.8 K. According to Kirklin (2000) this may be due to salicylic acid impurities, which produces a drastic lowering of the melting temperature of acetylsalicylic acid. Moreover, as can be seen in Fig. 1 (lidocaine + thymol or coumarin), experimental temperatures measured by different techniques (DSC - light blue filled symbols or melting points device - dark blue filled symbols) are in good agreement.

Analyzing Fig. 1 it can be seen that the studied systems involving coumarin are, in general, characterized by a single eutectic point and show small deviations from the ideal SLE curves, excepting coumarin + ibuprofen, where significant positive deviations to thermodynamic ideality are observed in the coumarin rich side. These systems have a eutectic temperature and composition similar to the predicted values considering ideality (Table S3), as further supported by the activity coefficients estimated from the experimental data, which are very close to 1 - Table S1 and Figure S1. This means that the molecular interactions between coumarin and the studied APIs are similar to those found in the liquid phases of the pure components. While the coumarin molecule presents a hydrogen bond acceptor character, all the APIs investigated present both donor and acceptor sites the latter being dominant. It is not then expected to observe strong interactions in the binary mixtures, which are very close to those present in the pure compounds as the structural features and functional groups in both are quite similar. This has been also observed before for mixtures of menthol and monocarboxylic acids (Abranches et al., 2020; Alhadid et al., 2020; Martins et al., 2018; Verma and Banerjee, 2018), lidocaine (Corvis et al., 2010; Kang et al., 2000), ibuprofen (Kang et al., 2004; Stott et al., 1998), alcohols and hydrocarbons (Okuniewski et al., 2017, 2016), or ubiquinone (Sun et al., 2019). Regarding the COSMO-RS predictions, this tool was able to successfully describe the SLE of the mixtures of coumarin + APIs with an average absolute deviation between experimental and predicted temperatures by COSMO-RS of $4.3 \pm 2.9 \mathrm{~K}$.

Despite the ideal behavior and the fact that these are not deep eutectic systems, the results reported for mixtures containing coumarin still show a quite significant temperature depression. At the eutectic point, the temperature is 23 and $104 \mathrm{~K}$ lower than the API melting point for systems with ibuprofen and acetaminophen, respectively.

When changing the lactone coumarin for the terpenoid thymol, negative deviations to ideality were observed close to the eutectic point and in the thymol rich side for the systems with carboxylic acids: acetylsalicylic acid, ibuprofen, and ketoprofen, indicating that thymol has stronger interactions with these APIs than coumarin. Thymol is a strong hydrogen bond donor (Abranches et al., 2019b) what may lead to favorable interactions between the two compounds. However, these interactions are not strong enough to lead to significant temperature depressions compared to those predicted by the ideal mixture model Table S3. COSMO-RS is not fully able to describe the non-ideal behavior of these mixtures containing thymol. Average absolute deviations of $14.5 \pm 13.3,7.1 \pm 5.7$ and $8.2 \pm 5.7 \mathrm{~K}$ were obtained between the experimental and predicted temperatures for systems containing thymol, and acetylsalicylic acid, ibuprofen, or ketoprofen, respectively.

At this point one must highlight the peculiar shape of the phase diagrams of thymol and carboxylic acids as expressed by the significant change of the carboxylic acid activity coefficient with the composition (Figure S1). In the literature, several works report that pharmaceuticals 

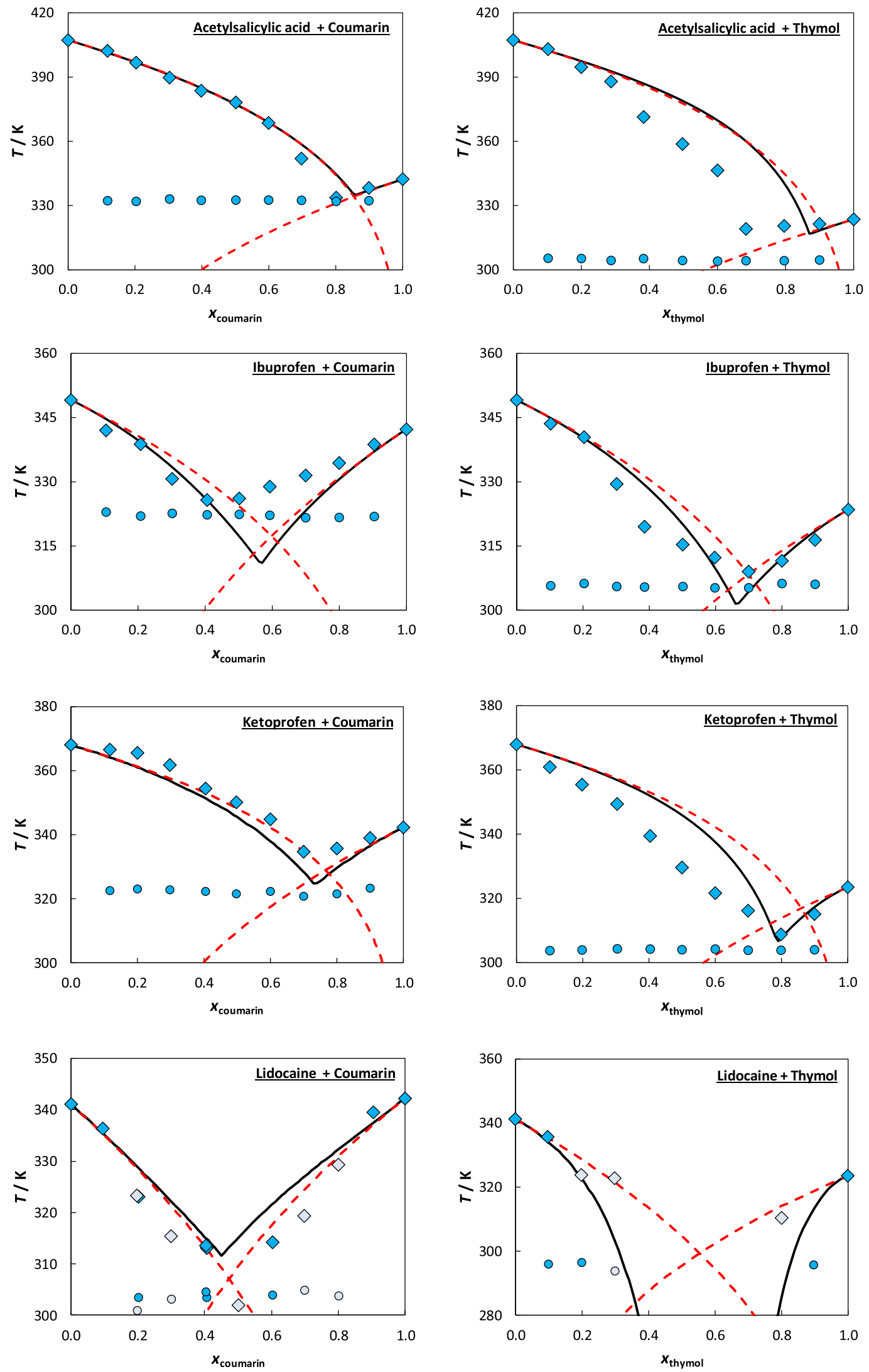

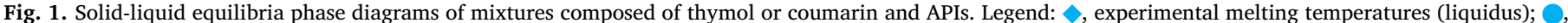

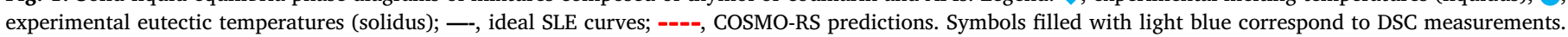



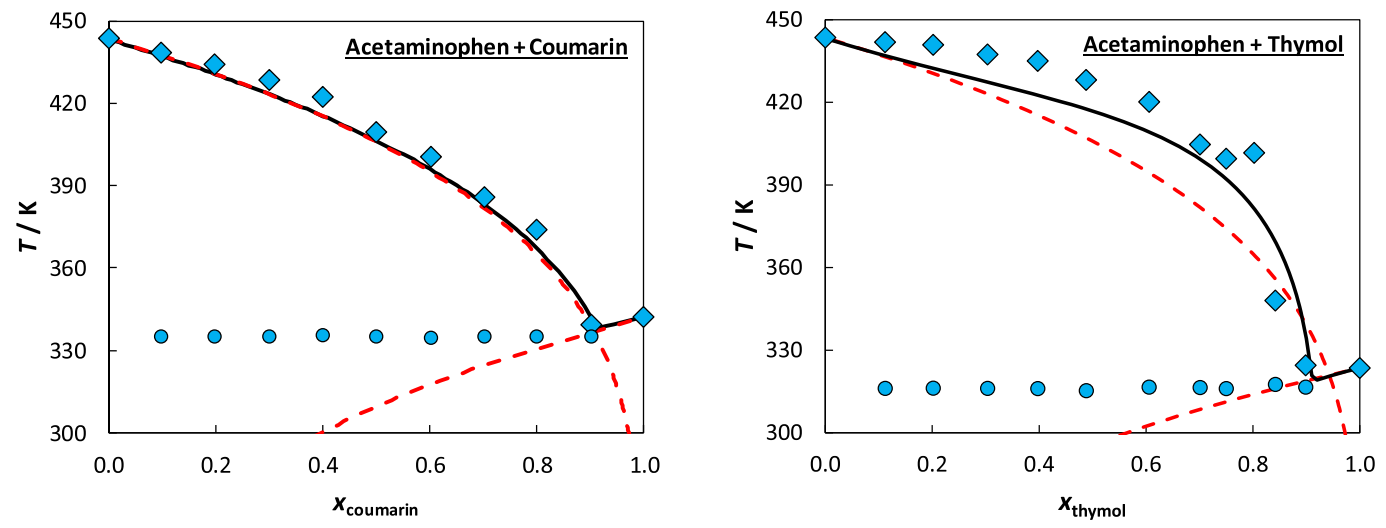

Fig. 1. (continued)

investigated here may exhibit different crystal structures (Dichi et al., 2018; Dudognon et al., 2008; Shohin et al., 2012). However, that only occur's at very specific conditions, as happens for racemic ibuprofen for

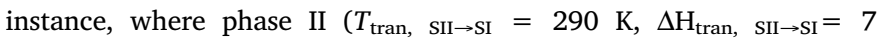
$\mathrm{kJ} \cdot \mathrm{mol}^{-1}$ ) is metastable and conditioned by a quench to 60 degrees below Tg (Dudognon et al., 2013, 2008). Regarding acetylsalicylic acid, an elusive form II was considered unstable by many until Bond et al. (2011) were able to obtain single crystals by introducing acetylsalicylic acid anhydride during the crystallization of acetylsalicylic acid in organic solvents. DSC measurements performed from $293.15 \mathrm{~K}$ to melting reveal thermal events in acetylsalicylic acid + thymol and ibuprofen + thymol at 368 and $318 \mathrm{~K}$, respectively, but not evidencing any isothermal reversible solid phase transformations.

The system thymol + acetaminophen shows positive deviations from ideality, indicating that the interactions between the excipient and API are not favorable. These deviations happen because thymol is a weak hydrogen bond acceptor (Abranches et al., 2019b), increasing with the amount of thymol in the mixture and are satisfactorily predicted by COSMO-RS. A color change on the mixture thymol + acetaminophen was observed after some days, suggesting that the mixture is unstable, and that some reaction may have occurred. The instability of the mixture is one of the undesirable effects on drug pre-formulation, and it must be considered.

In the system thymol + lidocaine, only a few experimental points are given due to difficulties in crystallizing the sample. Often the mixtures with thymol undergo supercooling, being hard to induce their crystallization until the glass transition. The crystallization was usually only observed upon heating after several heating-cooling cycles, yet on some occasions, not even this technique induces sample crystallization; which was also observed by Wolbert et al. (2019) for systems containing lidocaine. Nevertheless, despite the limited information measured for this system, its eutectic point seems to be close to the equimolar composition and the system nearly ideal. Here, COSMO-RS fails in predicting the SLE phase diagram. Similar to a few other systems studied before (Abranches et al., 2019a), here the curves do not intersect as the change of the activity coefficients with composition at low temperatures is unsatisfactory.

Mixtures involving thymol show more significant temperature depressions (compared to the melting temperature of the pure API) than mixtures involving coumarin, demonstrating its liquefying ability, and negative deviations to ideality when in mixture with carboxylic acids. However, the attribution of the designation deep eutectic systems to thymol + acetylsalicylic acid, ibuprofen, or ketoprofen is questionable since the deviations are in general not very strong, and these mixtures only present negative deviations from ideality in one side of their phase diagrams. Overall the reported mixtures involving thymol or coumarin, are hydrophobic which limits or prevents some applications, but also opens room for other pharmaceutical applications for example in dermal administrations (Kang et al., 2001; Patel et al., 2007; Roberts et al., 1977).

A few systems reported in this work were previously described in the literature. Kang et al. (2001) reported that the system thymol + lidocaine forms homogenous oils at $298.15 \mathrm{~K}$ within the range $0.30-0.70$ (lidocaine mole fraction). In this work, the mixtures prepared at compositions between 0.30 and 0.60 (lidocaine mole fraction) also yield liquids at room temperature. However, as can be seen by the SLE phase diagram in Fig. 1, the system thymol + lidocaine seems to follow an ideal behavior with a measured eutectic temperature close to $295 \mathrm{~K}$. This means that in the mentioned composition range, the mixtures are metastable and in a supercooled state where the liquid phase is maintained at a temperature inferior to the real melting temperature. Wolbert et al. (2019) measured two points of the system thymol + lidocaine - Figure S2 - at $x_{\text {thymol }}=0.2$ and 0.8 . The former seems to correspond to the solidus (and not liquidus) line, whereas the later perfectly matches the melting temperature measured in this work. Stott et al. (1998) and Wolbert et al. (2019), reported the SLE phase diagram of thymol + ibuprofen - Figure S3. Both sets of data are in good agreement with the ones measure in this work for the solidus and liquidus lines.

\subsection{Ionic excipients - quaternary ammonium salts}

Aiming to explore the impact of the excipient nature (ionic vs. nonionic) on the eutectic formation, the results presented above were further compared with mixtures involving $[\mathrm{Ch}] \mathrm{Cl},\left[\mathrm{N}_{1111}\right] \mathrm{Cl}$, or $\left[\mathrm{N}_{4444}\right] \mathrm{Cl}$ and the same APIs, i.e., type III eutectic systems.

Fig. 2 shows the SLE phase diagrams and activity coefficients of mixtures of thymol, coumarin, or [Ch] $\mathrm{Cl}$ and acetylsalicylic acid, ibuprofen, ketoprofen, lidocaine, or acetaminophen. Additionally, for ibuprofen and acetaminophen, the SLE phase diagrams with $\left[\mathrm{N}_{1111}\right] \mathrm{Cl}$, and $\left[\mathrm{N}_{4444}\right] \mathrm{Cl}$ are also presented. Data on $[\mathrm{Ch}] \mathrm{Cl}$ and acetylsalicylic acid, ibuprofen, ketoprofen, or acetaminophen were taken from our previous work (Abranches et al., 2019a). The remaining systems were measured in this work, and data are listed in Tables S1 and S2 of Supporting Information.

Other authors previously studied some of these systems. In their search for liquid pharmaceuticals, Abbott et al. (2017) investigated eutectic mixtures composed of $[\mathrm{Ch}] \mathrm{Cl}$ and several other compounds commonly used in pharmaceutical formulations, including acetylsalicylic acid and acetaminophen. For $[\mathrm{Ch}] \mathrm{Cl}+$ acetaminophen, a eutectic temperature of $314 \mathrm{~K}$ at equimolar composition was reported (Abbott et al., 2017). This value is in fair agreement with the one measured by us (Abranches et al., 2019a), $321 \mathrm{~K}$ at the same composition. The mixture $[\mathrm{Ch}] \mathrm{Cl}+$ acetylsalicylic acid is reported by some authors (Abbott et al., 2017; Aroso et al., 2016) to be liquid at room temperature. However, Abranches et al. (2019a) reported the complete 
phase diagram and the eutectic point to be $340 \mathrm{~K}$ at a mole fraction of $30 \%$ of $[\mathrm{Ch}] \mathrm{Cl}$.

It is very interesting to note that despite $[\mathrm{Ch}] \mathrm{Cl}$ high melting point (597 K) (Fernandez et al., 2017) and when mixed with the above mentioned APIs, the prototypical DES-forming component [Ch] Cl forms liquid mixtures at temperatures close to the body temperature $(310 \mathrm{~K})$ at the eutectic point. These temperature depressions are usually attributed to the strength of the hydrogen bond established between [Ch] Cl and the API (Abbott et al., 2017, 2003). Thus, its ionic character does not seem to be a disadvantage in the formation of the mixtures despite the non-ionic character of the studied APIs. On the other side, mixtures of thymol and coumarin with the same APIs lead to much weaker temperature depressions when compared to the non-ionic excipient melting point, which is also much lower $\left(T_{\mathrm{m} \text {,thymol }}=323.5 \mathrm{~K}\right.$ and $T_{\mathrm{m} \text {,coumarin }}=342.3 \mathrm{~K}$ ).
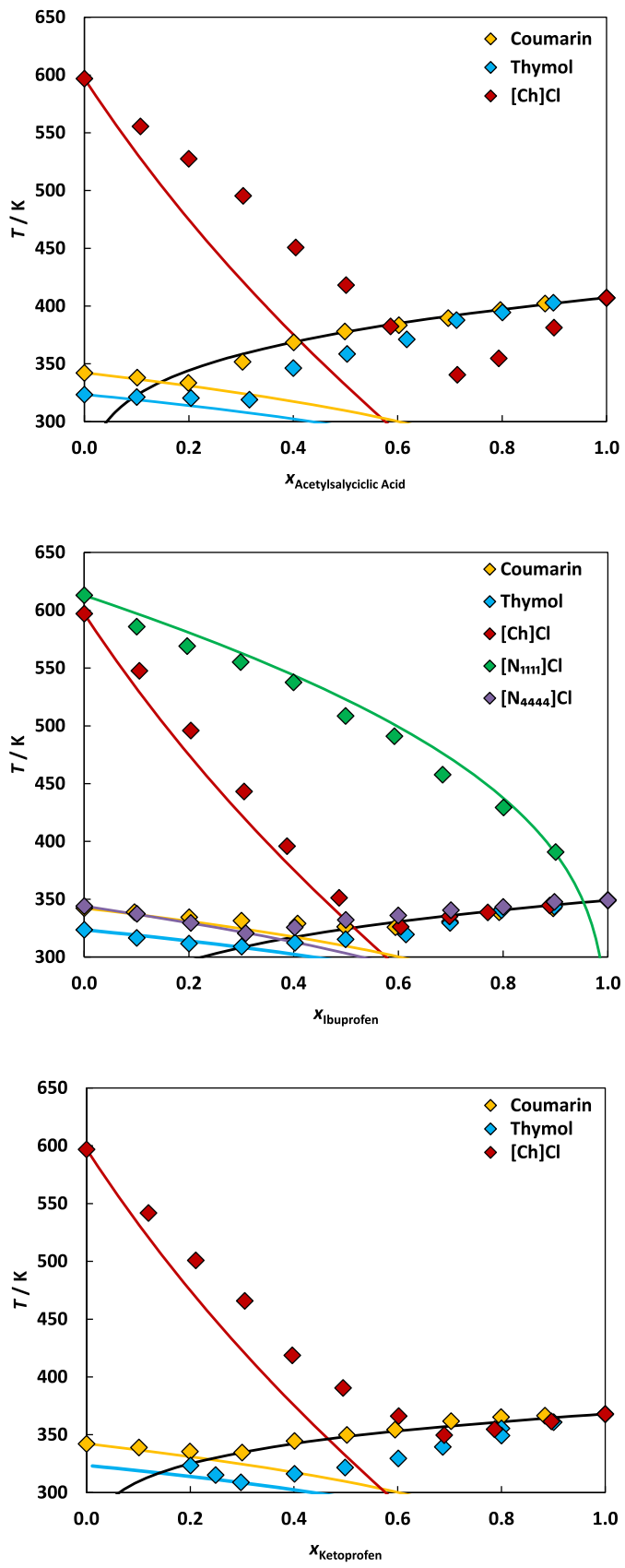

By analyzing the activity coefficients - Fig. 2 - the solubility curve of $[\mathrm{Ch}] \mathrm{Cl}$ shows positive deviations from ideality in most systems, and a quasi-ideal behavior in the case of $[\mathrm{Ch}] \mathrm{Cl}+$ acetaminophen. Thus, as observed in other works (Abranches et al., 2020; Crespo et al., 2017), the prototypical hydrogen bond acceptor $[\mathrm{Ch}] \mathrm{Cl}$ is unable to induce negative deviations to ideality, reaffirming in this work its inability to establish strong interactions in the studied mixtures. On the opposite, in the API rich side, strong negative deviations from ideality are observed, which is a behavior observed in many other systems (Crespo et al., 2018; Silva et al., 2019), and suggests that the melting point depression of the mixture is favored by small quantities of [Ch]Cl. An exception is the system $[\mathrm{Ch}] \mathrm{Cl}+$ lidocaine, where positive deviations from ideality are observed in the API rich side too.

By replacing the cation choline with tetramethylammonium the results are somewhat different. As can be seen by the activity
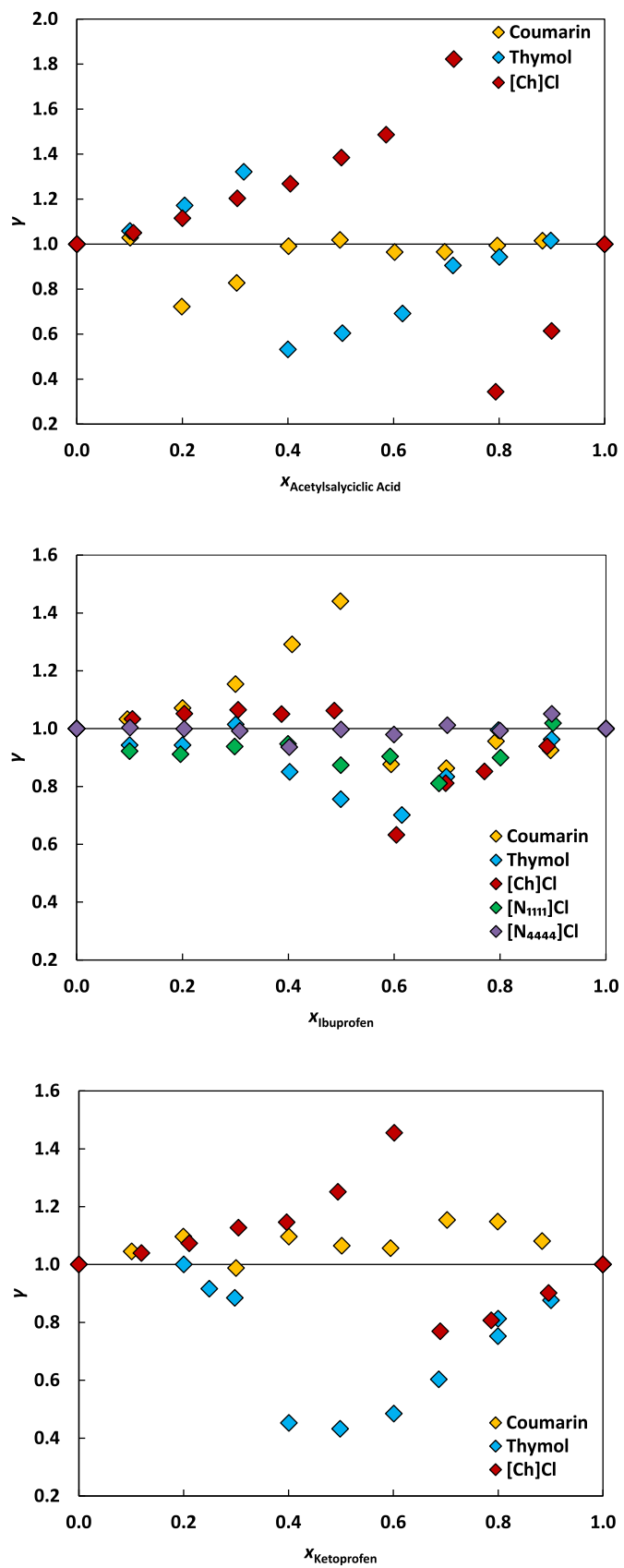

Fig. 2. Solid-liquid equilibria phase diagrams (left) of mixtures composed of thymol, coumarin, $[\mathrm{Ch}] \mathrm{Cl}$ (Abranches et al., 2019a), [ $\left.\mathrm{N}_{1111}\right] \mathrm{Cl}$ or [ $\left.\mathrm{N}_{4444}\right] \mathrm{Cl}$ and $\mathrm{APIs}$, and the correspondent activity coefficients (right). Symbols correspond to experimental data while solid lines correspond to the ideal solubility curves (left) and ideality (right). 

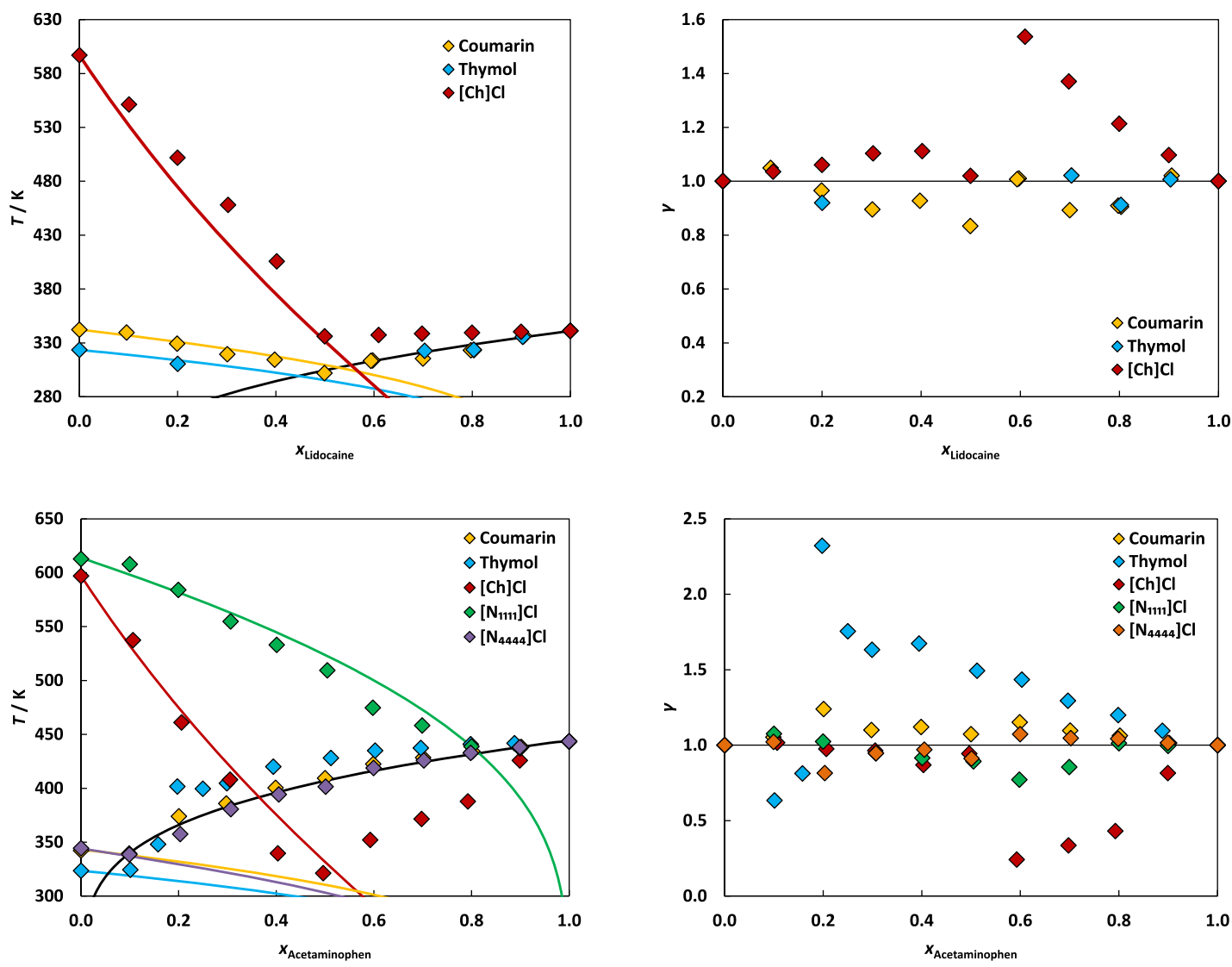

Fig. 2. (continued)

coefficients displayed in Fig. 2, mixtures of ibuprofen, or acetaminophen, with $\left[\mathrm{N}_{1111}\right] \mathrm{Cl}$ result in solubility curves with mild deviations from ideality. This means that, in opposition to what was observed previously (Abranches et al., 2020), the replacement of the hydroxyethyl group of the choline cation by a methyl group in $\left[\mathrm{N}_{1111}\right] \mathrm{Cl}$, does not induce stronger interactions and negative deviations from ideality in these systems. Nonetheless, in mixture with ibuprofen, $[\mathrm{Ch}] \mathrm{Cl}$ shows positive deviations to ideality meaning weaker interactions than in its pure phase, while $\left[\mathrm{N}_{1111}\right] \mathrm{Cl}$ has stronger interactions with the API resulting in small negative deviations.

In mixtures involving $\left[\mathrm{N}_{4444}\right] \mathrm{Cl}$, the activity coefficients are approximately 1 on both sides of the phase diagram. In the ammonium rich side, the same behavior was also observed in mixtures of $\left[\mathrm{N}_{4444}\right] \mathrm{Cl}+$ fatty acids or fatty alcohols (Abranches et al., 2020) and $\left[\mathrm{N}_{1111}\right] \mathrm{Cl}+\left[\mathrm{N}_{4444}\right] \mathrm{Cl}$ (Abranches et al., 2019c). However, while in those cases $\left[\mathrm{N}_{4444}\right] \mathrm{Cl}$ behaves as a chloride donating agent inducing negative deviations to ideality in the alcohols/acid side, here an ideal behavior is observed in the API rich side.

As observed in other studies, [Ch]Cl is not able to establish strong interactions and form deep eutectic systems with the APIs investigated. And, even removing its hydroxyl group, $\left[\mathrm{N}_{1111}\right] \mathrm{Cl}$ and $\left[\mathrm{N}_{4444}\right] \mathrm{Cl}$ are also not good candidates to be used as liquefying agents of the mentioned APIs. The observed behavior is a direct consequence of the structural complexity of the APIs herein studied. In general, by using ammonium chlorides as liquefying agents, smaller temperature depressions (compared to the melting temperature of the pure API) than the ones obtained with non-ionic excipients were achieved.

\section{Conclusions}

In this work, mixtures involving potential ionic or non-ionic excipients and APIs were investigated by measuring the SLE phase diagrams in the full range of compositions. In general, the studied systems involving coumarin are quasi-ideal, while in some systems, small negative deviations from ideality were observed when using thymol. The predictive model COSMO-RS was used to describe the mixtures SLE and a good quantitative description of the structural effects of different excipients and APIs in the SLE of the studied systems was obtained. Therefore, these results suggest that COSMO-RS can be used as a priori tool for the design of novel eutectic mixtures combining pharmaceuticals and nonionic hydrophobic components. The impact of the excipient nature (ionic or non-ionic) on the eutectic formation, was evaluated by measuring the SLE phase diagrams of the same APIs with $[\mathrm{Ch}] \mathrm{Cl},\left[\mathrm{N}_{1111}\right] \mathrm{Cl}$ or $\left[\mathrm{N}_{4444}\right] \mathrm{Cl}$. The melting temperature depression (compared to the API melting temperature) of systems involving nonionic excipients is more significant than when using ionic ones meaning that the eutectic is favored by type V eutectic systems. These studies can be useful in the design of new eutectic mixtures involving APIs that can lead to their liquefaction by a eutectic formation and thus to the development of more efficient drug delivery systems.

\section{CRediT authorship contribution statement}

Mónia A.R. Martins: Conceptualization, Data curation, Formal analysis, Methodology, Supervision, Writing - review \& editing. Liliana P. Silva: Data curation, Methodology. Patrícia S. Jorge: Data curation, Formal analysis, Methodology, Writing - review \& editing. Dinis $\mathbf{O}$. Abranches: Conceptualization, Data curation, Formal analysis. Simão P. Pinho: Conceptualization, Formal analysis, Funding acquisition, Supervision, Writing - review \& editing. João A.P. Coutinho: Conceptualization, Formal analysis, Funding acquisition, Supervision, Writing - review \& editing.

\section{Acknowledgments}

This work was developed within the scope of the project CICECO- 
Aveiro Institute of Materials, UIDB/50011/2020 \& UIDP/50011/2020, and CIMO-Mountain Research Center, UIDB/00690/2020, both financed by national funds through the Portuguese Foundation for Science and Technology/MCTES. L.P.S. acknowledges FCT for her PhD grant (SFRH/BD/135976/2018).

\section{Supplementary materials}

Supplementary material associated with this article can be found, in the online version, at doi:10.1016/j.ejps.2020.105583.

\section{References}

Abbott, A.P., Ahmed, E.I., Prasad, K., Qader, I.B., Ryder, K.S., 2017. Liquid pharmaceuticals formulation by eutectic formation. Fluid Phase Equilib 448, 2-8. https://doi. org/10.1016/j.fluid.2017.05.009.

Abbott, A.P., Capper, G., Davies, D.L., Rasheed, R.K., Tambyrajah, V., 2003. Novel solvent properties of choline chloride/urea mixtures. Chem. Commun. 99, 70-71. https:// doi.org/10.1039/b210714g.

Abranches, D.O., Larriba, M., Silva, L.P., Melle-Franco, M., Palomar, J.F., Pinho, S.P., Coutinho, J.A.P., 2019a. Using COSMO-RS to design choline chloride pharmaceutical eutectic solvents. Fluid Phase Equilib 497, 71-78. https://doi.org/10.1016/j.fluid. 2019.06.005.

Abranches, D.O., Martins, M.A.R., Silva, L.P., Schaeffer, N., Pinho, S.P., Coutinho, J.A.P., 2019b. Phenolic hydrogen bond donors in the formation of non-ionic deep eutectic solvents: the quest for type V DES. Chem. Commun. 55, 10253-10256. https://doi. org/10.1039/C9CC04846D.

Abranches, D.O., Martins, R.O., Silva, L.P., Martins, M.A.R., Pinho, S.P., Coutinho, J.A.P., 2020. Liquefying compounds by forming deep eutectic solvents: a case study for organic acids and alcohols. J. Phys. Chem. B 124, 4174-4184. https://doi.org/10. 1021/acs.jpcb.0c02386.

Abranches, D.O., Schaeffer, N., Silva, L.P., Martins, M.A.R., Pinho, S.P., Coutinho, J.A.P., 2019c. The role of charge transfer in the formation of type i deep eutectic solventanalogous ionic liquid mixtures. Molecules 24, 3687. https://doi.org/10.3390/ molecules24203687.

Alhadid, A., Mokrushina, L., Minceva, M., 2020. Design of deep eutectic systems: a simple approach for preselecting eutectic mixture constituents. Molecules 25, 1077-1088. https://doi.org/10.3390/molecules25051077.

Aroso, I.M., Silva, J.C., Mano, F., Ferreira, A.S.D., Dionísio, M., Sá-Nogueira, I., Barreiros, S., Reis, R.L., Paiva, A., Duarte, A.R.C., 2016. Dissolution enhancement of active pharmaceutical ingredients by therapeutic deep eutectic systems. Eur. J. Pharm. Biopharm. 98, 57-66. https://doi.org/10.1016/j.ejpb.2015.11.002.

Berg, J.M., Stryer, L., Tymoczko, J.L., 2010. Biochemistry, 7th ed. W.H. Freeman.

Bond, A.D., Solanko, K.A., Parsons, S., Redder, S., Boese, R., 2011. Single crystals of aspirin form II: crystallisation and stability. Cryst. Eng. Comm. 13, 399-401. https:// doi.org/10.1039/C0CE00588F.

Brown, M.B., Martin, G.P., Jones, S.A., Akomeah, F.K., 2006. Dermal and transdermal drug delivery systems: current and future prospects. Drug Deliv. 13, 175-187. https://doi.org/10.1080/10717540500455975.

Cherukuvada, S., Nangia, A., 2014. Eutectics as improved pharmaceutical materials: design, properties and characterization. Chem. Commun. 50, 906-923. https://doi. org/10.1039/C3CC47521B.

Corvis, Y., Négrier, P., Lazerges, M., Massip, S., Léger, J.M., Espeau, P., 2010. Lidocaine/ 1 -menthol binary system: cocrystallization versus solid-state immiscibility. J. Phys. Chem. B 114, 5420-5426. https://doi.org/10.1021/jp101303j.

Coutinho, J.A.P., Andersen, S.I., Stenby, E.H., 1995. Evaluation of activity coefficient models in prediction of alkane solid-liquid equilibria. Fluid Phase Equilib 103, 23-39. https://doi.org/10.1016/0378-3812(94)02600-6.

Crespo, E.A., Silva, L.P., Martins, M.A.R., Bülow, M., Ferreira, O., Sadowski, G., Held, C., Pinho, S.P., Coutinho, J.A.P., 2018. The role of polyfunctionality in the formation of [Ch]Cl-carboxylic acid-based deep eutectic solvents. Ind. Eng. Chem. Res. 57, 11195-11209. https://doi.org/10.1021/acs.iecr.8b01249.

Crespo, E.A., Silva, L.P., Martins, M.A.R., Fernandez, L., Ortega, J., Ferreira, O., Sadowski, G., Held, C., Pinho, S.P., Coutinho, J.A.P., 2017. Characterization and modeling of the liquid phase of deep eutectic solvents based on fatty acids/alcohols and choline chloride. Ind. Eng. Chem. Res. 56, 12192-12202. https://doi.org/10.1021/acs.iecr. $7 \mathrm{~b} 02382$.

Dichi, E., Sghaier, M., Guiblin, N., 2018. Reinvestigation of the paracetamol-caffeine, aspirin-caffeine, and paracetamol-aspirin phase equilibria diagrams. J. Therm. Anal. Calorim. 131, 2141-2155. https://doi.org/10.1007/s10973-017-6855-6.

Duarte, A.R.C., Ferreira, A.S.D., Barreiros, S., Cabrita, E., Reis, R.L., Paiva, A., 2017. A comparison between pure active pharmaceutical ingredients and therapeutic deep eutectic solvents: solubility and permeability studies. Eur. J. Pharm. Biopharm. 114, 296-304. https://doi.org/10.1016/j.ejpb.2017.02.003.

Dudognon, E., Correia, N.T., Danède, F., Descamps, M., 2013. Solid-solid transformation in racemic Ibuprofen. Pharm. Res. 30, 81-89. https://doi.org/10.1007/s11095-0120851-0.

Dudognon, E., Danède, F., Descamps, M., Correia, N.T., 2008. Evidence for a new crystalline phase of racemic Ibuprofen. Pharm. Res. 25, 2853-2858. https://doi.org/10. 1007/s11095-008-9655-7.

Eckert, F., Klamt, A., 2002. Fast solvent screening via quantum chemistry: COSMO-RS approach. AIChE J. 48, 369-385. https://doi.org/10.1002/aic.690480220.
Elliott, J.R., Lira, C.T., 2012. Introductory Chemical Engineering Thermodynamics, 2nd editio, ed. Prentiche Hall.

Fernandez, L., Silva, L.P., Martins, M.A.R., Ferreira, O., Ortega, J., Pinho, S.P., Coutinho, J.A.P., 2017. Indirect assessment of the fusion properties of choline chloride from solid-liquid equilibria data. Fluid Phase Equilib 448, 9-14. https://doi.org/10.1016/ j.fluid.2017.03.015.

Gala, U., Pham, H., Chauhan, H., 2013. Pharmaceutical applications of eutectic mixtures. J. Dev. Drugs 2, 1-2. https://doi.org/10.4172/2329-6631.1000e130.

Jun, H.W., Kang, L., 1999. Enhanced transdermal anesthesia of local anesthetic agents. WO2000069471A1.

Kalasz, H., Antal, I., 2006. Drug Excipients. Curr. Med. Chem. 13, 2535-2563. https:// doi.org/10.2174/092986706778201648.

Kalepu, S., Nekkanti, V., 2015. Insoluble drug delivery strategies: review of recent advances and business prospects. Acta Pharm. Sin. B 5, 442-453. https://doi.org/10. 1016/J.APSB. 2015.07.003.

Kang, L., Jun, H.., Mani, N., 2001. Preparation and characterization of two-phase melt systems of lidocaine. Int. J. Pharm. 222, 35-44. https://doi.org/10.1016/S03785173(01)00689-5.

Kang, L., Jun, H.W., McCall, J.W., 2000. Physicochemical studies of lidocaine-menthol binary systems for enhanced membrane transport. Int. J. Pharm. 206, 35-42. https:// doi.org/10.1016/S0378-5173(00)00505-6.

Kang, L., Park, M.O., Jun, H.W., 2004. Two-phase melt systems of ibuprofen for enhanced membrane permeation. Pharm. Dev. Technol. 9, 349-357. https://doi.org/10.1081/ PDT-200032991.

Kirklin, D.R., 2000. Enthalpy of combustion of acetylsalicylic acid. J. Chem. Thermodyn. 32, 701-709. https://doi.org/10.1006/jcht.1999.0650.

Klamt, A., 2005. COSMO-RS From Quantum Chemistry to Fluid Phase Thermodynamics and Drug Design. Elsevier, Amsterdam, The Netherlands.

Lazerges, M., Rietveld, I.B., Corvis, Y., Céolin, R., Espeau, P., 2010. Thermodynamic studies of mixtures for topical anesthesia: lidocaine-salol binary phase diagram. Thermochim. Acta 497, 124-128. https://doi.org/10.1016/j.tca.2009.08.016.

Martins, M.A.R., Crespo, E.A., Pontes, P.V.A., Silva, L.P., Bülow, M., Maximo, G.J., Batista, E.A.C., Held, C., Pinho, S.P., Coutinho, J.A.P., 2018. Tunable hydrophobic eutectic solvents based on terpenes and monocarboxylic acids. ACS Sustain. Chem. Eng. 6, 8836-8846. https://doi.org/10.1021/acssuschemeng.8b01203.

Martins, M.A.R., Pinho, S.P., Coutinho, J.A.P., 2019. Insights into the nature of eutectic and deep eutectic mixtures. J. Sol. Chem. 48, 962-982. https://doi.org/10.1007/ s10953-018-0793-1.

Matos, M.A.R., Sousa, C.C.S., Miranda, M.S., Morais, V.M.F., Liebman, J.F., 2009. Energetics of coumarin and chromone. J. Phys. Chem. B 113, 11216-11221. https:// doi.org/10.1021/jp9026942.

Maxwell, R., Chickos, J., 2012. An examination of the thermodynamics of fusion, vaporization, and sublimation of ibuprofen and naproxen by correlation gas chromatography. J. Pharm. Sci. 101, 805-814. https://doi.org/10.1002/jps.22803.

Mohammadi-Samani, S., Yousefi, G., Mohammadi, F., Ahmadi, F., 2014. Meloxicam transdermal delivery: effect of eutectic point on the rate and extent of skin permeation. Iran. J. Basic Med. Sci. 17, 112-118. https://doi.org/10.22038/ijbms.2014. 2243.

Mota, F.L., Carneiro, A.P., Queimada, A.J., Pinho, S.P., Macedo, E.A., 2009. Temperature and solvent effects in the solubility of some pharmaceutical compounds: Measurements and modeling. Eur. J. Pharm. Sci. 37, 499-507. https://doi.org/10. 1016/j.ejps.2009.04.009.

Okuniewski, M., Paduszyński, K., Domańska, U., 2017. Phase diagrams in representative terpenoid systems: measurements and calculations with leading thermodynamic models. Ind. Eng. Chem. Res. 56, 9753-9761. https://doi.org/10.1021/acs.iecr. $7 \mathrm{~b} 02207$.

Okuniewski, M., Paduszyński, K., Domańska, U., 2016. (Solid + liquid) equilibrium phase diagrams in binary mixtures containing terpenes: new experimental data and analysis of several modelling strategies with modified UNIFAC (Dortmund) and PC-SAFT equation of state. Fluid Phase Equilib 422, 66-77. https://doi.org/10.1016/j.fluid. 2015.12.048.

Patel, T., Ishiuji, Y., Yosipovitch, G., 2007. Menthol: a refreshing look at this ancient compound. J. Am. Acad. Dermatol. 57, 873-878. https://doi.org/10.1016/j.jaad. 2007.04.008.

Pedro, S.N., Freire, M.G., Freire, C.S.R., Silvestre, A.J.D., 2019. Deep eutectic solvents comprising active pharmaceutical ingredients in the development of drug delivery systems. Expert Opin. Drug Deliv. 16, 497-506. https://doi.org/10.1080/17425247. 2019.1604680.

Pontes, P.V.A., Crespo, E.A., Martins, M.A.R., Silva, L.P., Neves, C.M.S.S., Maximo, G.J., Hubinger, M.D., Batista, E.A.C., Pinho, S.P., Coutinho, J.A.P., Sadowski, G., Held, C., 2017. Measurement and PC-SAFT modeling of solid-liquid equilibrium of deep eutectic solvents of quaternary ammonium chlorides and carboxylic acids. Fluid Phase Equilib 448, 69-80. https://doi.org/10.1016/j.fluid.2017.04.007.

Prausnitz, J.M., Lichtenthaler, R.N., Azevedo, E.G., 1970. Molecular thermodynamics of fluid-phase equilibria. J Chem. Thermodyn. https://doi.org/10.1016/0021-9614(70) 90078-9.

Roberts, M.S., Anderson, R.A., Swarbrick, J., 1977. Permeability of human epidermis to phenolic compounds. J. Pharm. Pharmacol. 29, 677-683. https://doi.org/10.1111/j. 2042-7158.1977.tb11434.x.

Sangster, J., 1999. Phase diagrams and thermodynamic properties of binary systems of drugs. J. Phys. Chem. Ref. Data 28, 889-930. https://doi.org/10.1063/1.556040.

Savjani, K.T., Gajjar, A.K., Savjani, J.K., 2012. Drug solubility: importance and enhancement techniques. ISRN Pharm. 2012, 1-10. https://doi.org/10.5402/2012/ 195727.

Schwarz, J., Weisspapir, M., 2005. Vehicle for topical delivery of anti-inflammatory compounds. EP1858556A1. 
Shohin, I.E., Kulinich, J.I., Ramenskaya, G.V., Abrahamsson, B., Kopp, S., Langguth, P., Polli, J.E., Shah, V.P., Groot, D.W., Barends, D.M., Dressman, J.B., 2012. Biowaiver monographs for immediate-release solid oral dosage forms: ketoprofen. J. Pharm. Sci. 101, 3593-3603. https://doi.org/10.1002/jps.23233.

Silva, L.P., Araújo, C.F., Abranches, D.O., Melle-Franco, M., Martins, M.A.R., Nolasco, M.M., Ribeiro-Claro, P.J.A., Pinho, S.P., Coutinho, J.A.P., 2019. What a difference a methyl group makes-probing choline-urea molecular interactions through urea structure modification. Phys. Chem. Chem. Phys. 21, 18278-18289. https://doi.org/ 10.1039/c9cp03552d.

Smith, E.L., Abbott, A.P., Ryder, K.S., 2014. Deep eutectic solvents (DESs) and their applications. Chem. Rev. 114, 11060-11082. https://doi.org/10.1021/cr300162p.

Stott, P.W., Williams, A.C., Barry, B.W., 1998. Transdermal delivery from eutectic systems: enhanced permeation of a model drug, ibuprofen. J. Control. Release 50, 297-308. https://doi.org/10.1016/S0168-3659(97)00153-3.

Strickley, R.G., Oliyai, R., 2007. Formulation challenges of prodrugs, in: prodrugs. Biotechnology: Pharmaceutical Aspects. Springer, New York. https://doi.org/10. 1007/978-0-387-49785-3_30.

Sun, M., Shichao, D., Tang, W., Jia, L., Gong, J., 2019. Design of spherical crystallization for drugs based on thermal-induced liquid-liquid phase separation: case studies of water-insoluble drugs. Ind. Eng. Chem. Res. 58, 20401-20411. https://doi.org/10. 1021/acs.iecr.9b03795.

Tiwari, G., Tiwari, R., Sriwastawa, B., Bhati, L., Pandey, S., Pandey, P., Bannerjee, S.K., 2012. Drug delivery systems: an updated review. Int. J. Pharm. Investig 2, 2-11. https://doi.org/10.4103/2230-973x.96920.

TURBOMOLE V7.1 2016, A development of university of Karlsruhe and Forschungszentrum karlsruhe GmbH, 1989-2007, TURBOMOLE GmbH, since 2007; available from http://www.turbomole.com.

Verma, R., Banerjee, T., 2018. Liquid-liquid extraction of lower alcohols using mentholbased hydrophobic deep eutectic solvent: experiments and COSMO-SAC predictions. Ind. Eng. Chem. Res. 57, 3371-3381. https://doi.org/10.1021/acs.iecr.7b05270.

Wassvik, C.M., Holmén, A.G., Bergström, C.A.S., Zamora, I., Artursson, P., 2006. Contribution of solid-state properties to the aqueous solubility of drugs. Eur. J. Pharm. Sci. 29, 294-305. https://doi.org/10.1016/j.ejps.2006.05.013.

Wolbert, F., Brandenbusch, C., Sadowski, G., 2019. Selecting excipients forming therapeutic deep eutectic systems-a mechanistic approach. Mol. Pharm. 16, 3091-3099. https://doi.org/10.1021/acs.molpharmaceut.9b00336. 